\title{
Cubic Splines and Approximate Solution of Singular Integral Equations*
}

\author{
By Erica Jen and R. P. Srivastav**
}

\begin{abstract}
Of concern here is the numerical solution of singular integral equations of Cauchy type; i.e., equations involving principal value integrals. The unknown function is expressed as the product of an appropriate weight function and a cubic spline. The problem is reduced to a system of linear algebraic equations which is solved for the approximate values of the function at the knots. An estimate is provided for the maximum error of the approximate solution. Numerical results from the spline method are compared with those obtained using other methods.
\end{abstract}

1. Introduction. In the numerical solution of singular integral equations of the form

$$
a(s) g(s)+\frac{b(s)}{\pi} \int_{-1}^{1} \frac{g(t) d t}{t-s}+\int_{-1}^{1} K(t, s) g(t) d t=f(s), \quad-1<s<1,
$$

it is of ten desirable to avoid the techniques of regularization and to adopt instead a direct method for computation. One such method proposed by Erdogan and Gupta [2] replaces the unknown function $g(t)$ by

$$
\phi(t) w(t)
$$

[where $w(t)$ is the weight function determined by the index theory], and uses a Gaussian integration formula for the numerical evaluation of the integral expression. Although the method appears to be accurate for equations with well-behaved kernels and input functions, the inherent restrictions on the choice of node and collocation points could prove to be a handicap in some situations.

As an alternative to Gauss-Chebyshev methods, Gerasoulis and Srivastav [4] proposed that the function $\phi(t)$ in (1.2) be approximated using piecewise linear functions. This procedure permits the analytical evaluation of the integral expressions, and affords flexibility in the choice of node and collocation points. The present paper extends the results of [4] and of Gerasoulis [3] with the development of a cubic spline approximation method. In addition to providing higher accuracy, the spline method can also be expected to be applicable to the numerical solution of singular integro-differential equations.

The organization of the paper is as follows: Section 2 describes a procedure for the solution of $(1.1)$ with $a(s)=0$ and $b(s)=1$, Section 3 contains error analysis

Received August 8, 1980; revised January 30, 1981.

1980 Mathematics Subject Classification. Primary 45E05, 41 A15.

Key words and phrases. Spline approximation, numerical solution of singular integral equations.

* The results of this paper were presented at the Twenty-sixth Conference of Army Mathematicians (June 1980).

** Sponsored by the United States Army under Contract No. DAAG-29-80-C-0030. 0025-5718/81/0000-0162/\$02.75 
results, and Section 4 provides comparisons of numerical results obtained from various methods.

2. Reduction to a Linear Algebraic System. Consider the case where $a(s)=0$, and $b(s)=1$ in (1.1), and the solution is known to possess square root singularities at \pm 1 . (The method described below is applicable in general, although in some cases it may be necessary to evaluate certain integrals numerically.) Set

$$
g(t)=\phi(t)\left(1-t^{2}\right)^{-1 / 2} \text {. }
$$

In most applications, it is possible to exploit the symmetry properties of the problem and to work with either odd or even functions. Therefore, assume the number of node points to be $(2 n+1)$, and let $-1=t_{0}<t_{1}<\cdots<t_{2 n}=1$. Replace the unknown function $\phi(t)$ by splines $S(t)=S_{j}(t)(j=1,2, \ldots, 2 n)$ on the interval $\left[t_{j-1}, t_{j}\right]$. It is computationally convenient to use the form [1]

$$
\begin{aligned}
S_{j}(t)= & \frac{M_{j-1}}{6 h_{j}}\left(t_{j}-t\right)^{3}+\frac{M_{j}}{6 h_{j}}\left(t-t_{j-1}\right)^{3}+\left(\frac{\phi_{j}}{h_{j}}-\frac{M_{j} h_{j}}{6}\right)\left(t-t_{j-1}\right) \\
& +\left(\frac{\phi_{j-1}}{h_{j}}-\frac{M_{j-1} h_{j}}{6}\right)\left(t_{j}-t\right), \quad j=1,2, \ldots, 2 n,
\end{aligned}
$$

where $h_{j}=t_{j}-t_{j-1}, \phi_{j}=\phi\left(t_{j}\right)$, and $M_{j}=S_{j}^{\prime \prime}\left(t_{j}\right)=S_{j-1}^{\prime \prime}\left(t_{j}\right)$. (Although the above expressions for $S_{j}(t)$ involve the moments, or second derivatives, of splines, it is possible to use instead their first derivatives.) The function $K\left(t, s_{k}\right)$ is approximated by $K_{j}\left(t, s_{k}\right)$ using a cubic interpolation formula in each of the intervals $\left[t_{j-1}, t_{j}\right]$. In this way, the original equation is replaced by a discrete analogue

$$
\sum_{j=1}^{2 n} \int_{t_{j-1}}^{t_{j}} \frac{S_{j}(t) d t}{\sqrt{1-t^{2}}\left(t-s_{k}\right)}+\sum_{j=1}^{2 n} \int_{t_{j-1}}^{t_{j}} \frac{S_{j}(t) K_{j}\left(t, s_{k}\right) d t}{\sqrt{1-t^{2}}}=f\left(s_{k}\right),
$$

where the collocation points are chosen so that $t_{k-1}<s_{k}<t_{k}$. All the quantities in (2.4) can be evaluated analytically to yield $2 n$ linear equations for the $(4 n+2)$ unknowns $M_{0}, M_{1}, \ldots, M_{2 n}, \phi_{0}, \phi_{1}, \ldots, \phi_{2 n}$. An additional $(2 n-1)$ equations are furnished by the continuity of the derivatives of splines; namely,

$$
\begin{aligned}
M_{j-1}+2 M_{j}\left(\frac{h_{j}+h_{j+1}}{h_{j}}\right)+\frac{h_{j+1}}{h_{j}} M_{j+1}=\frac{6}{h_{j}}\left(\frac{\phi_{j+1}-\phi_{j}}{h_{j+1}}-\frac{\phi_{j}-\phi_{j-1}}{h_{j}}\right), \\
j=1,2, \ldots, 2 n-1 .
\end{aligned}
$$

Two equations relating the values of the moments at the endpoints are needed. These equations are usually chosen to be of the form

$$
\alpha_{0} M_{0}+\beta_{0} M_{1}=C_{0}, \quad \beta_{2 n} M_{2 n-1}+\alpha_{2 n} M_{2 n}=C_{2 n} .
$$

Finally, a single equation is obtained from the compatibility condition

$$
\sum_{j=1}^{2 n} \int_{t_{j-1}}^{t_{j}} \frac{S_{j}(t) d t}{\sqrt{1-t^{2}}}=k, \quad k \text { constant. }
$$

Thus a total of $(4 n+2)$ equations in as many variables is obtained. The coefficient matrix for the system of equations is of the form

$$
\left(\begin{array}{l}
A^{1} \\
A^{2} \\
A^{3}
\end{array}\right)
$$


where $A^{1}$ is the $2 n \times(4 n+2)$ submatrix of coefficients obtained from the integral equation evaluated at the $2 n$ collocation points;

$A^{2}$ is the $1 \times(4 n+2)$ submatrix of coefficients obtained from the compatibility condition;

$A^{3}$ is the $(2 n+1) \times(4 n+2)$ submatrix of coefficients obtained from the moments conditions and the continuity relations for splines.

In order to display the elements of the coefficient matrix in convenient form, some operator notation is needed. Define operators $I_{k}, J_{k}$ by

$$
\left(I_{k} f\right)(s)=\int_{t_{k-1}}^{t_{k}} \frac{f(t) d t}{\sqrt{1-t^{2}}(t-s)}, \quad J_{k} f=\int_{t_{k-1}}^{t_{k}} \frac{f(t) d t}{\sqrt{1-t^{2}}} .
$$

Note that for polynomial functions $f$, the expressions for $\left(I_{k} f\right)(s)$ and $J_{k} f$ can be evaluated analytically. In particular,

$$
\begin{aligned}
\left(I_{k} t^{p}\right)(s)= & \int_{t_{k-1}}^{t_{k}} \frac{t^{p-1}}{\sqrt{1-t^{2}}} d t+s \int_{t_{k-1}}^{t_{k}} \frac{t^{p-2}}{\sqrt{1-t^{2}}} d t+\cdots \\
& +s^{p-1} \int_{t_{k-1}}^{t_{k}} \frac{d t}{\sqrt{1-t^{2}}}+s^{p}\left(I_{k} 1\right)(s), \quad p=1,2, \ldots,
\end{aligned}
$$

and $[5$, p. 147]

$$
\left(I_{k} 1\right)(s)=\frac{1}{\sqrt{1-s^{2}}} \ln \left|\frac{\left\{-1+\sqrt{1-s^{2}}+s \tan \frac{\theta_{k}}{2}\right\} \tan \frac{\theta_{k-1}}{2}+s-\left(\sqrt{1-s^{2}}+1\right) \tan \frac{\theta_{k}}{2}}{\left\{-1-\sqrt{1-s^{2}}+s \tan \frac{\theta_{k}}{2}\right\} \tan \frac{\theta_{k-1}}{2}+s-\left(1-\sqrt{1-s^{2}}\right) \tan \frac{\theta_{k}}{2}}\right|,
$$

where $\theta_{k}=\arcsin t_{k}$. Then the elements of the submatrix $A^{1}$ are given for $j=$ $1,2, \ldots, 2 n$ by

$$
\begin{aligned}
& A_{j, i}^{1}=\left.I_{i}\left[\frac{1}{6 h_{i}}\left(t_{i}-t\right)^{3}-\frac{h_{i}}{6}\left(t_{i}-t\right)\right]\right)\left(s_{j}\right) \\
&+J_{i}\left[\frac{1}{6 h_{i}}\left(t_{i}-t\right)^{3} K_{i}\left(t, s_{j}\right)-\frac{h_{i}}{6}\left(t_{i}-t\right) K_{i}\left(t, s_{j}\right)\right] \\
&+J_{i}\left[\frac{1}{6 h_{i}}\left(t_{i}-t\right)^{3} K_{i}\left(t, s_{j}\right)-\frac{h_{i}}{6}\left(t_{i}-t\right) K_{i}\left(t, s_{j}\right)\right] \\
&+\left(I_{i-1}\left[\frac{1}{6 h_{i-1}}\left(t-t_{i-2}\right)^{3}-\frac{h_{i-1}}{6}\left(t-t_{i-2}\right)\right]\right)\left(s_{j}\right) \\
&+J_{i-1}\left[\frac{1}{6 h_{i-1}}\left(t-t_{i-2}\right)^{3} K_{i-1}\left(t, s_{j}\right)-\frac{h_{i-1}}{6}\left(t-t_{i-2}\right) K_{i-1}\left(t, s_{j}\right)\right], \\
&=\left(I_{i}\left[\frac{1}{h_{i}}\left(t_{i}-t\right)\right]\right)\left(s_{j}\right)+J_{i}\left[\left(t_{i}-t\right) K_{i}\left(t, s_{j}\right)\right] \\
&+\left(I_{i-1}\left[\frac{1}{h_{i-1}}\left(t-t_{i-2}\right)\right]\right)\left(s_{j}\right)+J_{i-1}\left[\left(t-t_{i-2}\right) K_{i-1}\left(t, s_{j}\right)\right], 2 n+1, \\
& \quad i=2 n+2,2 n+3, \ldots, 4 n+2,
\end{aligned}
$$


where $\left(I_{k} f\right)(s)$ and $J_{k} f$ are taken to be zero for $k=0,2 n+1$. The elements of $A^{2}$ are given by

$$
\begin{aligned}
A_{1, i}^{2} & =J_{i}\left[\frac{1}{6 h_{i}}\left(t_{i}-t\right)^{3}-\frac{h_{i}}{6}\left(t_{i}-t\right)\right]+J_{i-1}\left[\frac{1}{6 h_{i-1}}\left(t-t_{i-2}\right)^{3}-\frac{h_{i-1}}{6}\left(t-t_{i-2}\right)\right], \\
& i=1,2, \ldots, 2 n+1, \\
& =J_{i}\left[\frac{1}{h_{i}}\left(t_{i}-t\right)\right]+J_{i-1}\left[\frac{1}{h_{i-1}}\left(t-t_{i-2}\right)\right], \quad i=2 n+2,2 n+3, \ldots, 4 n+2,
\end{aligned}
$$

where again $J_{k} f=0$ for $k=0,2 n+1$.

The elements of $A^{3}$ are given by

$$
\begin{aligned}
& A_{1, j}=\alpha_{0}, \quad i=1, \quad A_{2 n+1, i}=\beta_{2 n}, \quad i=2 n, \\
& =\beta_{0}, \quad i=2, \quad=\alpha_{2 n}, \quad i=2 n+1, \\
& =0 \text {, otherwise, } \quad=0, \quad \text { otherwise, }
\end{aligned}
$$

and for $j=2,3, \ldots, 2 n$ by

$$
\begin{aligned}
A_{j, 1} & =1, \quad i=j-1, \\
& =2\left(\frac{h_{j}+h_{j-1}}{h_{j-1}}\right), \quad i=j, \\
& =\frac{h_{j}}{h_{j-1}}, \quad i=j+1, \\
& =\frac{6}{h_{j} h_{j-1}}, \quad i=2 n+j, \\
& =\frac{6}{h_{j} h_{j-1}}-\frac{6}{h_{j}^{2}}, \quad i=2 n+j+1, \\
& =\frac{6}{h_{j}^{2}}, \quad i=2 n+j+2, \\
& =0, \quad \text { otherwise. }
\end{aligned}
$$

From the above, it can be seen that the coefficient matrix $A$ has the structure

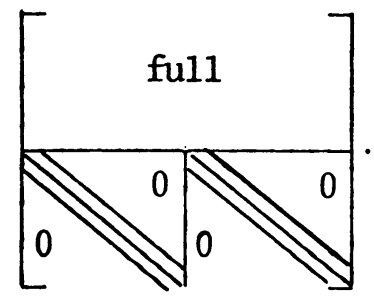

Note that, when solving the system by Gaussian elimination using only partial pivoting, it may be advisable to rearrange the matrix so that the $\phi_{j}$ 's are computed first, thus reducing the effect of round-off error propagation.

3. Error Analysis. Define the functions $\phi^{*}, \phi^{e}$ as follows:

(i) $\phi^{*}$ is the Type II cubic spline on the true values $\phi\left(t_{i}\right), i=0,1, \ldots, 2 n$, with $\phi^{* \prime \prime}\left(t_{0}\right)=\phi^{\prime \prime}\left(t_{0}\right), \phi^{* \prime \prime}\left(t_{2 n}\right)=\phi^{\prime \prime}\left(t_{2 n}\right)$; 
(ii) $\phi^{\varepsilon}$ is the spline on the computed values for $\phi\left(t_{i}\right), i=0,1, \ldots, 2 n$. Let

$$
\begin{aligned}
\mathbf{x} & =\left[\phi^{\prime \prime}\left(t_{0}\right), \phi^{\prime \prime}\left(t_{1}\right), \ldots, \phi^{\prime \prime}\left(t_{2 n}\right), \phi\left(t_{0}\right), \phi\left(t_{1}\right), \ldots, \phi\left(t_{2 n}\right)\right], \\
\mathbf{x}^{*} & =\left[\phi^{* \prime \prime}\left(t_{0}\right), \phi^{* \prime \prime}\left(t_{1}\right), \ldots, \phi^{* \prime \prime}\left(t_{2 n}\right), \phi^{*}\left(t_{0}\right), \phi^{*}\left(t_{1}\right), \ldots, \phi^{*}\left(t_{2 n}\right)\right], \\
\mathbf{x}^{\varepsilon} & =\left[\phi^{\varepsilon \prime \prime}\left(t_{0}\right), \phi^{\varepsilon \prime \prime}\left(t_{1}\right), \ldots, \phi^{\varepsilon \prime \prime}\left(t_{2 n}\right), \phi^{\varepsilon}\left(t_{0}\right), \phi^{\varepsilon}\left(t_{1}\right), \ldots, \phi^{e}\left(t_{2 n}\right)\right],
\end{aligned}
$$

and

$$
\mathbf{f}=\left[f\left(s_{1}\right), f\left(s_{2}\right), \ldots, f\left(s_{2 n}\right), k, C_{0}, 0, \ldots, 0, C_{2 n}\right] .
$$

It will be assumed below that the splines used are the natural splines, so $C_{0}=C_{2 n}$ $=0$. The system of equations which is being solved can therefore be represented as

$$
A \mathbf{x}^{\varepsilon}=\mathbf{f} \text {. }
$$

The vector $\mathbf{x}$ of true values satisfies

$$
A \mathbf{x}=\tilde{\mathbf{f}}
$$

where $\tilde{\mathbf{f}}=\mathbf{f}+\boldsymbol{\theta}$, and $\boldsymbol{\theta}$ is the vector of errors in the numerical integration induced by the use of splines. Hence

$$
\left\|\mathbf{x}^{e}-\mathbf{x}\right\| \leqslant\left\|A^{-1}\right\| \cdot\|\theta\|
$$

Note that $A$ depends on the spline knots and the collocation points. The above inequality can be used to obtain an error estimate for the spline method. The components of $\boldsymbol{\theta}$ are given by

$$
\begin{aligned}
\theta_{j}=\frac{1}{\pi} \int_{-1}^{1} \frac{\left(\phi^{*}-\phi\right) d t}{\left(t-s_{j}\right) \sqrt{1-t^{2}}}+\int_{-1}^{1} \frac{\left(K_{e} \phi^{*}-K \phi\right) d t}{\sqrt{1-t^{2}}}, \\
j=1,2, \ldots, 2 n,
\end{aligned}
$$

where $K_{e}$ is the piecewise cubic approximant to $K$, and

$$
\begin{aligned}
\theta_{j} & =\int_{-1}^{1} \frac{\left(\phi^{*}-\phi\right) d t}{\sqrt{1-t^{2}}}, \quad j=2 n+1 \\
& =\phi^{\prime \prime}\left(t_{0}\right), \quad j=2 n+2 \\
& =\phi^{\prime \prime}\left(t_{2 n}\right), \quad j=4 n+2 \\
& =0, \quad \text { otherwise. }
\end{aligned}
$$

Let

$$
\varepsilon(t)=\phi^{*}-\phi .
$$

Then the second term in (3.4) is easily shown to be bounded by

$$
\pi\left\{\max _{-1<t<1}\left|\phi^{*}\right| \cdot \max \left|K_{\varepsilon}-K\right|+\max |\varepsilon| \cdot \max |K|\right\},
$$

which, using the results of [6], is $O\left(h^{4}\right)$ for $h=\max _{j} h_{j}$. Furthermore, again using the results of [6], the first term in (3.4) can be shown to be $O\left(h^{7 / 2-\delta}\right), \delta>0$. Hence the maximum error of the spline method is given by (3.3) with $\|\theta\|$ of order $h^{7 / 2-\delta}$.

In practice, it has been found that for problems with known solutions, the spline method produces results considerably more accurate than predicted by (3.3), thus indicating that the error bounds above could probably be significantly improved. 
4. Numerical Results. The spline method has been used to solve a number of singular integral equations, including the following:

Example 1.

(4.1) $\frac{1}{\pi} \int_{-1}^{1} \frac{g(t) d t}{t-s}+\frac{1}{\pi} \int_{-1}^{1} \sin (t-s) g(t) d t=J_{1}(1) \cos s+1, \quad-1<s<1$,

where $J_{1}$ is the Bessel function of the first kind of order 1. The solution $g(t)$ is required to satisfy the compatibility condition

$$
\int_{-1}^{1} g(t) d t=0
$$

Moreover, $g(t)$ is assumed to possess square-root singularities at \pm 1 , and hence to be expressible in the form

$$
g(t)=\phi(t)\left(1-t^{2}\right)^{-1 / 2}
$$

Then it can be seen that the true solution is given by (4.2) with $\phi(t)=t$. The table below displays the maximum error $\varepsilon$ in the values for $\phi(t)$ computed using the spline method.

$$
\begin{array}{cc}
\frac{n^{*}}{2} & \\
3 & \frac{\varepsilon}{000041} \\
4 & 0.000003 \\
& 0.000001
\end{array}
$$

For $n=7$, the computed solution was accurate to the limits of single-precision computation (8 digits).

${ }^{*} n$ is the number of nodes taken in the interval $[0,1)$.

Example 2.

$$
\frac{1}{\pi} \int_{-1}^{1} \frac{g(t)}{t-s} d t+\frac{1}{\pi} \int_{-1}^{1} \frac{t\left(t^{2}-s^{2}\right)}{\left(t^{2}+s^{2}\right)^{2}} g(t) d t=\sigma, \quad-1<s<1,
$$

subject to the compatibility condition

$$
\int_{-1}^{1} g(t) d t=0
$$

The above equation arises in the problem of a cruciform crack in an infinite isotropic elastic medium under constant load $\sigma$ along its four branches. As before, the function $g(t)$ is assumed to be of the form

$$
g(t)=\phi(t)\left(1-t^{2}\right)^{-1 / 2}
$$

The table below provides a comparison of results obtained from the ErdoganGupta method [2] (Column I), its Lobatto-Chebyshev variant [8] (Column II), and the spline method (Column III). 0.8636 is the value calculated by Rooke and Sneddon [7] and generally accepted for $\phi(1)$. 


\begin{tabular}{ccccc}
$\frac{n}{3}$ & Erdogan-Gupta & & Lobatto-Chebyshev & \\
\cline { 2 - 2 } 3 & 0.8364 & & & Spline \\
4 & 0.8388 & 0.8597 & 0.8846 \\
5 & 0.8629 & 0.8639 & 0.8641 \\
6 & 0.8638 & 0.8645 & 7.8638 \\
7 & 0.8653 & 0.8644 & 0.8637 \\
8 & 0.8628 & 0.8642 & 0.8636 \\
9 & 0.8650 & 0.8641 & 0.8636 \\
10 & 0.8628 & 0.8640 & 0.8636 \\
& & 0.8638 & 0.8636
\end{tabular}

Department of Mathematics and Statistics State University of New York at Stony Brook Long Island, New York 11794

1. J. H. Ahlberg, E. H. NiLson and J. L. WALSH, The Theory of Splines and Their Applications, Academic Press, New York, 1967.

2. F. ERDOGAN \& G. D. GuPTA, "On the numerical solution of singular integral equation," Quart. Appl. Math., v. 30, 1972, pp. 525-534.

3. A. Gerasoulis, Product Integration Methods for the Solution of Singular Integral Equations of Cauchy type, Dept. of Comput. Sci. Report No. DCS-TR-86 Rutgers Univ., New Brunswick, 1979.

4. A. Gerasoulis \& R. P. SRivastav, "A method for the numerical solution of singular integral equations with a principal value integral," Internat. J. Engrg. Sci. (To appear.)

5. I. S. Gradshteyn \& I. M. Ryzhix, Table of Integrals, Series, and Products, Academic Press, New York, 1965.

6. C. A. Hall \& W. W. MeYer “Optimal error bounds for cubic spline interpolation,” J. Approx. Theory, v. 16, 1976, pp. 105-122.

7. D. P. RoOKe \& I. N. SNEDDON, "The crack energy and stress intensity factor for a cruciform crack deformed by internal pressure," Internat. J. Engrg. Sci., v. 7, 1969, pp. 1079-1089.

8. P. S. TheOcaris \& N. I. Ionkmidis, "Numerical integration methods for the solution of singular integral equations," Quart Appl. Math., v. 35, 1977, pp. 173-183. 